\title{
CORRELAÇÃO LINEAR E ESPACIAL ENTRE A PRODUTIVIDADE DE FEIJÃO (Phaseolus vulgaris L) E DE ATRIBUTOS FíSICOS DE UM LATOSSOLO VERMELHO EM SELVÍRIA (MS, BRASIL) \\ LINEAR AND SPATIAL CORRELATION BETWEEN BEAN (Phaseolus vulgaris $L$ ) YIELD AND PHYSICAL ATTRIBUTES OF A RED LATOSOL IN SELVÍRIA (MS, BRAZIL)
}

\author{
RAFAEL MONTANARI ${ }^{1}$ \\ MOREL DE P. E CARVALHO1,* \\ GLÉCIO M. SIQUEIRA, ,** \\ JORGE DAFONTE DAFONTE ${ }^{32}$ \\ ANTONIO PAZ GONZÁLEZ ${ }^{4}$
}

\section{RESUMO}

O presente trabalho teve como objetivo avaliar a variabilidade espacial de atributos físicos do solo que envolve a relação massa/volume (macroporosidade, microporosidade, porosidade total e densidade do solo) e da cultura de feijão (produtividade de grãos) por meio da correlação linear e espacial entre tais atributos. As amostragens foram realizadas no ano agrícola de 2004/2005 na Fazenda Experimental de Ensino e Pesquisa da Faculdade de Engenharia/UNESP-Campus de llha

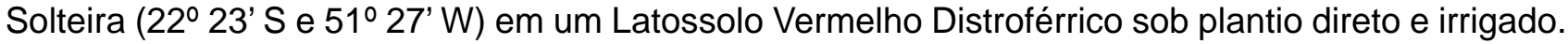
As amostras foram coletadas em 119 pontos de amostragem em uma área de 0,99 ha. A porosidade total (PT), macroporosidade (MA), microporosidade (MI), densidade do solo (DS) e a produtividade de grãos de feijão (PG) apresentaram baixa variabilidade, com alcances da dependência espacial entre 22,9 m e 77,9 m. A correlação linear entre os atributos do solo variou entre nula a alta. Entre a produtividade de grãos e os atributos físicos do solo a correlação linear foi nula.

PALAVRAS-CHAVES: Manejo do Solo, geoestatística, variabilidade espacial.

\footnotetext{
${ }^{1}$ Universidade Estadual Paulista, Campus de Ilha Solteira (UNESP) - Av. Brasil, 56, Centro, C. P. 31, 15385-000, Ilha Solteira, São Paulo, Brasil. *Autor para correspondência: E-mail: morel@agr.feis.unesp.br; rafamontana@hotmail.com.

${ }^{2}$ Instituto Agronômico (IAC), Centro de Pesquisa e Desenvolvimento de Solos e Recursos Agroambientais, Av. Barão de Itapura, 1481 CP28, 13020-970. Campinas, SP. ** Bolsista FAPESP. E-mail: glecio.machado@rai.usc.es.

${ }^{3}$ Universidad de Santiago de Compostela (USC), Escuela Politécnica Superior, 27002, Lugo, España. E-mail: jorge.dafonte@usc.es

${ }^{4}$ Universidad de A Coruña (UDC), Facultad de Ciencias, A Zapateira s/n, 15071 A Coruña, España. E-mail: tucho@udc.es.
} 


\begin{abstract}
:
This work aimed to evaluate the soil physical attributes spatial variability concerning to the relation mass/volume (macro porosity, micro porosity, total porosity, and soil density) and beans fields (gains yield) through the linear correlation and spatial variability analysis among cited attributes. Sampling was done at an experimental field of UNESP/Ilha Solteira, SP, Brazil on an Oxisol under no-tillage soil management and with irrigation during the cropping seasons of 2004/2005. The samples were collected in 119 different points in one area of 0.99 ha. The total soil porosity (PT), macro porosity (MA), micro porosity (MI), soil bulk density (SD) and beans yield (PG) showed low variability with spatial dependence ranges between $22.9 \mathrm{~m}$ and $77.9 \mathrm{~m}$. The linear correlation between the soil attributes ranged from null to high. Correlation between grain yield and the soil physical attributes was null.
\end{abstract}

KEYWORDS: Soil Management, geostatistics; spatial variability.

\section{INTRODUÇÃO}

No Brasil, o cultivo do feijoeiro (Phaseolus vulgaris L) constitui-se numa das principais explorações agrícolas, não só pela área cultivada, mas também pelo valor da produção. Todavia, os principais problemas com relação à baixa produtividade desta cultura então relacionados com a falta de cultivares mais produtivos (MEGDA et al., 2008) e adaptados as diferentes tipos de clima e solo (BOLLER \& CALDATO, 2001). Nesse sentido, nos últimos anos diversos autores tem se dedicado ao estudo da variabilidade espacial da produção de feijão e sua relação com atributos físicos do solo (FREDDI et al., 2005; SANTOS et al., 2005; MEGDA et al., 2008).

No sistema de semeadura direta, a qualidade física do solo é reduzida devido ao aumento da densidade do solo das camadas iniciais, com redução da porosidade superficial (BEUTLER et al., 2001). Na maioria dos casos, ocorre redução da macroporosidade e aumento da microporosidade e da densidade do solo (BEUTLER et al., 2001). A determinação do estado de compactação, da densidade e da porosidade do solo possibilita sugerir quais práticas, referentes ao seu manejo, poderão proporcionar maior produtividade e, por conseguinte, maior auto-sustentabilidade do ambiente agrícola (ALCÂNTARA \& FERREIRA, 2000). Neste aspecto, o mapeamento dos atributos físicos do solo torna-se de fundamental importância, sobretudo daqueles que possuem evidente correlação com a produtividade vegetal, entre os quais a macroporosidade, microporosidade, porosidade total e a densidade do solo (BENGOUGH et al., 2001).

A estatística clássica proporciona descrever analiticamente um determinado atributo do solo. Contudo, não leva em conta a localização espacial das amostras de solo e planta. Como a ação do sistema de preparo do solo não é uniforme em toda área, torna-se importante avaliar também a distribuição espacial da magnitude dos seus atributos. A geoestatística tem como base o semivariograma (TRANGMAR et al., 1985; WEBSTER \& OLIVER, 1990; VIEIRA, 1997), o qual permite a descrição da dependência espacial destes atributos. A análise geoestatística pode indicar alternativas de manejo, não só para reduzir os efeitos da variabilidade do solo sobre a produção das culturas, como também para aumentar a possibilidade de estimar respostas das culturas às determinadas práticas de manejo (TRANGMAR et al., 1985).

O objetivo deste trabalho, realizado num Latossolo Vermelho Distroférrico do Cerrado Brasileiro, semeado com a cultura do feijão irrigado, sob o sistema de semeadura direta foi analisar a variabilidade espacial de atributos físicos do solo que envolvem a relação massa/ volume (macroporosidade, microporosidade, 
porosidade total e densidade do solo) e da cultura de feijão (produtividade de grãos) por meio da correlação linear e espacial entre tais atributos.

\section{MATERIAL E MÉTODOS}

Este trabalho foi desenvolvido no ano agrícola 2004/05 em uma área de 0,99 ha manejada com semeadura direta irrigada com pivô central, na Fazenda de Ensino e Pesquisa da Faculdade de Engenharia - FE/UNESP, Campus de Ilha Solteira, localizada no município de Selvíria (MS, Brasil), na latitude $22^{\circ} 23^{\prime}$ S e na longitude $51^{\circ} 27^{\prime} \mathrm{W}$. De acordo com a classificação climática de Köppen o clima da região é do tipo Aw, caracterizado como tropical úmido com estação chuvosa no verão e seca no inverno, apresentando precipitação média anual de 1300 $\mathrm{mm}$ e temperatura média de $23,7^{\circ} \mathrm{C}$.

O solo da área de estudo é um Latossolo Vermelho distroférrico típico argiloso, A moderado (EMBRAPA, 2006). As amostras de solo e planta foram realizadas em 119 pontos de amostragem, sendo 44 pontos em uma malha regular de $15 \mathrm{~m}$ x $15 \mathrm{~m}$ e 75 pontos em uma segunda malha composta por 15 ilhas de amostragem com espaçamento de 7,5 m x 7,5 m (Figura 1).

No período da amostragem, o solo da área de estudo estava cultivado com feijão (Phaseolus vulgaris L) cultivar Pérola, com espaçamento entre linhas de $0,50 \mathrm{~m}$ e densidade de 15 sementes por metro na linha de semeadura.

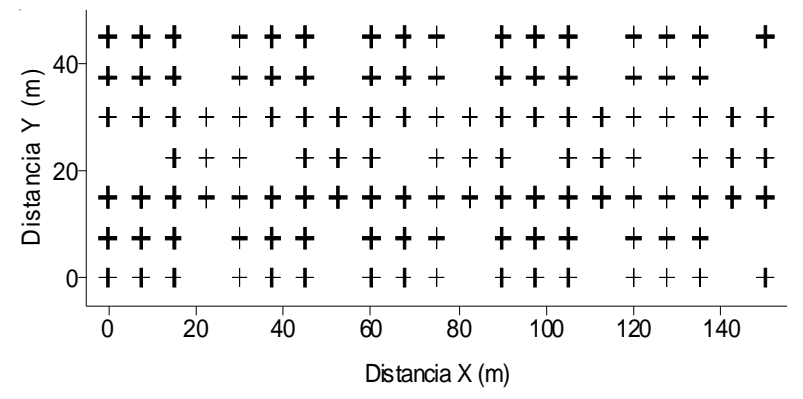

FIGURA 1. Grade de amostragem contendo 119 pontos, sendo 44 com espaçamento de $15 \mathrm{~m} \times 15$ $\mathrm{m}$ e 75 com espaçamento de $7,5 \mathrm{~m} \times 7,5 \mathrm{~m}$
As amostras de solo foram coletadas nas seguintes profundidades no dia 28/07/2004: 0,0$0,10,0,10-0,20,0,20-0,30$ e $0,30-0,40 \mathrm{~m}$. Os atributos físicos do solo amostrados foram: macroporosidade ( $\mathrm{MA}, \mathrm{m}^{3} \mathrm{~m}^{-3}$ ), microporosidade $\left(\mathrm{Ml}, \mathrm{m}^{3} \mathrm{~m}^{-3}\right)$, porosidade total $\left(\mathrm{PT}, \mathrm{m}^{3} \mathrm{~m}^{-3}\right)$ e a densidade do solo (DS, $\mathrm{kg} \mathrm{dm}^{-3}$ ). A produtividade ( $P G$, tha ${ }^{-1}$ ) da cultura de feijão (Phaseolus vulgaris L) foi determinada no dia 12/08/2004. Os atributos físicos do solo foram coletados utilizando anéis volumétricos de acordo com metodologia proposta por EMBRAPA (1997). A produtividade de grãos (PG) foi coletada nas condições de umidade de campo, numa área de $4,0 \mathrm{~m}^{2}$, contendo quatro linhas de plantas para todos os pontos amostrais. Seus valores foram transformados para as condições padronizadas de $0,13 \mathrm{~kg} \mathrm{~kg}^{-1}$ de umidade, sendo então representada em t ha-1.

O programa SAS (SCHLOTZHAVER \& LITTELL, 1997) foi utilizado para determinar os principais momentos estatísticos (média, valores mínimo e máximo, coeficiente de variação, assimetria e curtose), a normalidade dos dados por meio do teste de SHAPIRO \& WILK (1965) a $1 \%$ de probabilidade e a correlação linear simples entre os atributos de solo e planta. A análise geoestatística foi realizada utilizando o programa GS+ (ROBERTSON, 1998). Os atributos que apresentaram tendências nos seus dados foi aplicada a técnica do refinamento pela mediana, obtendo-se os respectivos resíduos. O ajuste do semivariograma, em função dos seus modelos, foi efetuado pela seleção inicial de: a) o maior coeficiente de determinação $\left(r^{2}\right)$; b) a menor soma dos quadrados dos desvios (RSS), e c) o maior valor de razão de dependência espacial entre amostras (RD). A decisão final do modelo representante do ajuste foi efetuada por meio da validação cruzada, assim como também para a definição do tamanho da vizinhança que proporcionou a melhor malha de krigagem. A razão de dependência espacial foi determinada de acordo com ROBERTSON (1998) conforme a seguinte expressão:

BioEng, Campinas, 3 (2): 169-179, mai/ago., 2009. 


$$
R D=\left(\frac{C_{1}}{C_{0}+C_{1}}\right) * 100
$$

onde: $R D$ é a razão de dependência espacial (\%); $\mathrm{C}_{0}$ é o efeito pepita; $\mathrm{C}_{1}$ é a variância estrutural, e $\mathrm{C}_{0}+\mathrm{C}_{1}$ é o patamar. Sendo, $\mathrm{RD}<25 \%$ baixa dependência espacial; RD entre $25-75 \%$ moderada dependência espacial e RD > $75 \%$ forte dependência espacial entre amostras.

\section{O software SURFER 7.0 (GOLDEN} SOFTWARE, 1999) foi utilizado para construção de mapas de isolinhas para os diferentes atributos envolvidos neste estudo, utilizando os valores estimados por meio da técnica de krigagem para os locais não amostrados.

\section{RESULTADOS E DISCUSSÃO}

A Tabela 1 apresenta os resultados da análise estatística. De acordo com GOMES \& GARCIA (2002) a variabilidade de um atributo pode ser classificada, considerando-se os valores de coeficiente de variação (CV), sendo: a) baixa, se menor do que $10 \%$; b) média, quando entre $10-20 \%$; c) alta, quando entre $20-30 \%$, e d) muito alta se maior do que $30 \%$. A Tabela 1 demonstra que a maioria dos atributos em estudo apresentam baixa variabilidade dos dados, exceto $\mathrm{MA}_{4}$ que apresentou variabilidade mediana. Estes resultados discordam de SOUZA et al. (2001), CARVALHO et al. (2002) e MEGDA et al. (2008) que encontraram media variabilidade para a macroporosidade do solo. Os demais atributos em estudo coincidem com MEGDA et al. (2008) que encontraram baixa variabilidade dos dados mesmo em profundidade para a microporosidade e a porosidade total. Com relação à produtividade de grãos de feijão (Phaseolus vulgaris $L$ ) FREDDI et al. (2005) e MEGDA et al. (2008) descrevem uma variabilidade mediana, enquanto que neste estudo a produtividade (PG) apresentou baixa variabilidade $(\mathrm{CV}=9,0 \%)$.

A macroporosidade nas camadas de 0,0$0,10 \mathrm{~m}$ de profundidade $\left(\mathrm{MA}_{1}\right), 0,10-0,20 \mathrm{~m}$ de profundidade $\left(\mathrm{MA}_{2}\right)$ e $0,2-0,3 \mathrm{~m}$ de profundidade
$\left(\mathrm{MA}_{3}\right)$ apresentaram distribuição de freqüência lognormal concordando com SOUZA et al. (2001) e CARVALHO et al. (2002). Os demais atributos apresentaram distribuição de freqüência normal por meio do teste de SHAPIRO \& WILK a $1 \%$ de probabilidade, concordando com SOUZA et al. (2001), CARVALHO et al. (2002) e MEGDA et al. (2008). A produtividade de grãos (PG) apresentou distribuição de freqüência normal coincidindo com FREDDI et al. (2005) e MEGDA et al. (2008). 
TABELA 1. Parâmetros estatísticos dos atributos de solo (porosidade total-PT, macroporosidade-MA, microporosidade-MI e densidade do solo-DS) e planta (produtividade de grãos-PG) estudados nas distintas profundidades

\begin{tabular}{|c|c|c|c|c|c|c|c|c|}
\hline \multirow[b]{2}{*}{$\begin{array}{c}\text { Atributo } \\
\text { s }\end{array}$} & \multirow[b]{2}{*}{$\begin{array}{c}\text { Unidad } \\
\mathrm{e}\end{array}$} & \multirow[b]{2}{*}{$\begin{array}{c}\text { Medi } \\
\text { a }\end{array}$} & \multicolumn{2}{|c|}{----- Valor ----- } & \multicolumn{3}{|c|}{-------- Coeficientes -------- } & \multirow[b]{2}{*}{ DF * } \\
\hline & & & $\begin{array}{c}\text { Mínim } \\
\text { o }\end{array}$ & $\begin{array}{c}\text { Máxim } \\
\text { o }\end{array}$ & $\begin{array}{c}\text { Variação } \\
(\%)\end{array}$ & $\begin{array}{c}\text { Curtos } \\
\text { e }\end{array}$ & $\begin{array}{l}\text { Assimetri } \\
\text { a }\end{array}$ & \\
\hline $\mathbf{P T}_{1}$ & & 0,434 & 0,363 & 0,519 & 7,7 & 0,480 & 0,282 & $0,171 \mathrm{n}$ \\
\hline $\mathbf{P T}_{2}$ & $m^{3} m^{-3}$ & 0,432 & 0,334 & 0,496 & 6,3 & 0,598 & $-0,304$ & $0,414 n$ \\
\hline $\mathbf{P T}_{3}$ & min in & 0,454 & 0,389 & 0,524 & 5,8 & 0,165 & 0,107 & $0,895 \mathrm{n}$ \\
\hline $\mathbf{P T}_{4}$ & & 0,494 & 0,426 & 0,546 & 4,9 & 0,196 & $-0,338$ & $\begin{array}{c}0,161 \mathrm{n} \\
0,208\end{array}$ \\
\hline $\mathbf{M A}_{1}$ & & 0,088 & 0,043 & 0,188 & 7,5 & 0,655 & 0,198 & $\begin{array}{c}\mathrm{Ln} \\
0,270\end{array}$ \\
\hline $\mathbf{M A}_{2}$ & $\mathrm{~m}^{3} \mathrm{~m}^{-3}$ & 0,090 & 0,040 & 0,168 & 6,2 & 0,039 & 0,017 & $\begin{array}{c}\mathrm{Ln} \\
0,682\end{array}$ \\
\hline $\mathbf{M A}_{3}$ & & 0,097 & 0,047 & 0,184 & 5,2 & 0,589 & 0,178 & Ln \\
\hline $\mathbf{M A}_{4}$ & & 0,143 & 0,073 & 0,212 & 17,5 & 0,385 & 0,302 & $0,330 \mathrm{n}$ \\
\hline $\mathbf{M I}_{1}$ & & 0,340 & 0,293 & 0,390 & 5,2 & 0,241 & $-0,094$ & $0,828 \mathrm{n}$ \\
\hline $\mathbf{M I}_{2}$ & & 0,339 & 0,303 & 0,375 & 4,5 & $-0,558$ & $-0,188$ & $0,234 \mathrm{n}$ \\
\hline $\mathrm{MI}_{3}$ & & 0,351 & 0,292 & 0,398 & 5,0 & 0,394 & $-0,093$ & $0,745 n$ \\
\hline $\mathbf{M I}_{4}$ & & 0,350 & 0,298 & 0,402 & 5,2 & 0,475 & $-0,238$ & $0,745 \mathrm{n}$ \\
\hline $\mathbf{D S}_{1}$ & & 1,491 & 1,230 & 1,646 & 5,6 & 0,084 & $-0,449$ & $0,448 \mathrm{n}$ \\
\hline $\mathbf{D S}_{2}$ & $\mathrm{ko} \mathrm{dm}^{-3}$ & 1,481 & 1,294 & 1,617 & 4,6 & 0,225 & $-0,218$ & $0,332 \mathrm{n}$ \\
\hline $\mathrm{DS}_{3}$ & $\mathrm{~kg} \mathrm{dm}$ & 1,400 & 1,251 & 1,557 & 4,4 & 0,057 & 0,185 & $0,493 \mathrm{n}$ \\
\hline $\mathrm{DS}_{4}$ & & 1,279 & 1,160 & 1,424 & 4,1 & 0,299 & 0,318 & $0,128 \mathrm{n}$ \\
\hline PG & $\mathrm{t} \mathrm{ha}^{-1}$ & 2,836 & 2,254 & 3,310 & 9,0 & 0,738 & 0,018 & $0,042 \mathrm{n}$ \\
\hline
\end{tabular}

*DF-Distribuição de freqüência por meio do teste de SHAPIRO \& WILK a $1 \%$ de probabilidade; Ln: dados com distribuição de freqüência não normal; n: dados com distribuição de freqüência normal

Os valores médios da macroporosidade (MA), presentes nas três primeiras profundidades, foram 0,085; 0,090 e 0,101 $\mathrm{m}^{3} \mathrm{~m}^{-3}$. Entretanto, na última profundidade $\left(\mathrm{MA}_{4}\right)$, seu valor médio foi de $0,143 \mathrm{~m}^{3} \mathrm{~m}^{-3}$. Desta forma, pode-se inferido que a $P G$ provavelmente venha a resultar um baixo valor. Isto por que, conforme preceitos de KIEHL (1979), nas três primeiras profundidades, onde se concentra a maior parte do sistema radicular do feijão, o solo apresentou-se compactado e, portanto, em desacordo com o que seria um solo tipicamente ideal. Já em relação a $\mathrm{MI}$, seus valores médios ficaram entre 0,340 $\left(\mathrm{MI}_{1}\right)$ e 0,351 $\left(\mathrm{MI}_{4}\right) \mathrm{m}^{3} \mathrm{~m}^{-3}$, ficando tal magnitude de forma semelhante às obtidas por SOUZA et al. (2001), CARVALHO et al. (2002) e MEGDA et al. (2008).
A PT apresentou valores médios entre 0,432 $\left(\mathrm{PT}_{1}\right)$ e $0,500\left(\mathrm{PT}_{4}\right) \mathrm{m}^{3} \mathrm{~m}^{-3}$, ficando, nesse caso, de forma semelhante às obtidas por SOUZA et al. (2001). Contudo, ficou ligeiramente menor do que a de CARVALHO et al. (2002), os quais observaram um valor de $0,55 \mathrm{~m}^{3} \mathrm{~m}^{-3}$. Por outro lado, a DS média, por apresentar valores que foram diminuindo em profundidade, com $1,49 \mathrm{~kg}$

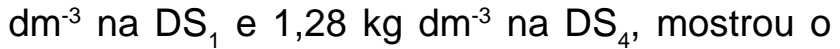
evidente estado de elevada compactação superficial no qual o solo trabalhado se encontrava. Tais valores foram semelhantes em magnitude àqueles obtidos por SOUZA et al. (2004). Distanciando-se valores encontrados por CARVALHO et al. (2002), nos quais ela possuiu menores valores. 
A produtividade média de grãos de feijão (PG, Tabela 1) foi de 2,836 $\mathrm{t} \mathrm{ha}^{-1}$, valor superior aos de FREDDI et al. (2005), SANTOS et al. (2005) e MEGDA et al. (2008), nos quais foram respectivamente de 2,650, 2,594, 2,210 e 2,752 $\mathrm{t}$ ha $^{-1}$. Segundo o critério de classificação da produtividade do feijão (FAHL et al., 1998), o elevado valor da produtividade média de grãos, observado no presente trabalho, atestou que a referida cultura apresentou relativa resistência ao estado de compactação no qual o do solo se encontrava, uma vez que sua DS, no geral, foi substancialmente alta na camada de 0,0-0,30 m, onde se concentra a maior parte do sistema radicular da cultura. Por outro lado, há que considerar que, tendo sido o feijão irrigado sob pivô central, o constante condicionamento do solo, em situações ideais de umidade, pôde ter suavizado o efeito deletério que a compactação poderia ter proporcionado para a diminuição de tal produtividade. Também, esse elevado valor da PG discordou da baixa produtividade esperada para solos compactados (com valores de macroporosidade inferiores a $0,100 \mathrm{~m}^{3} \mathrm{~m}^{-3}$ ), conforme preceitos estabelecidos para o solo ideal (BAVER et al., 1973; KIEHL, 1979). Vale ressaltar, que a produtividade média de grãos de feijão (PG) foi 4,5 maior que a média nacional $\left(0,630\right.$ t ha $\left.^{-1}\right)$ conforme relatado por NEHMI et al. (2003).

A Tabela 2 apresenta a matriz de correlação entre a produtividade de grãos de feijão e os atributos físicos do solo estudado.

TABELA 2. Matriz de correlação para os atributos de solo e planta estudados

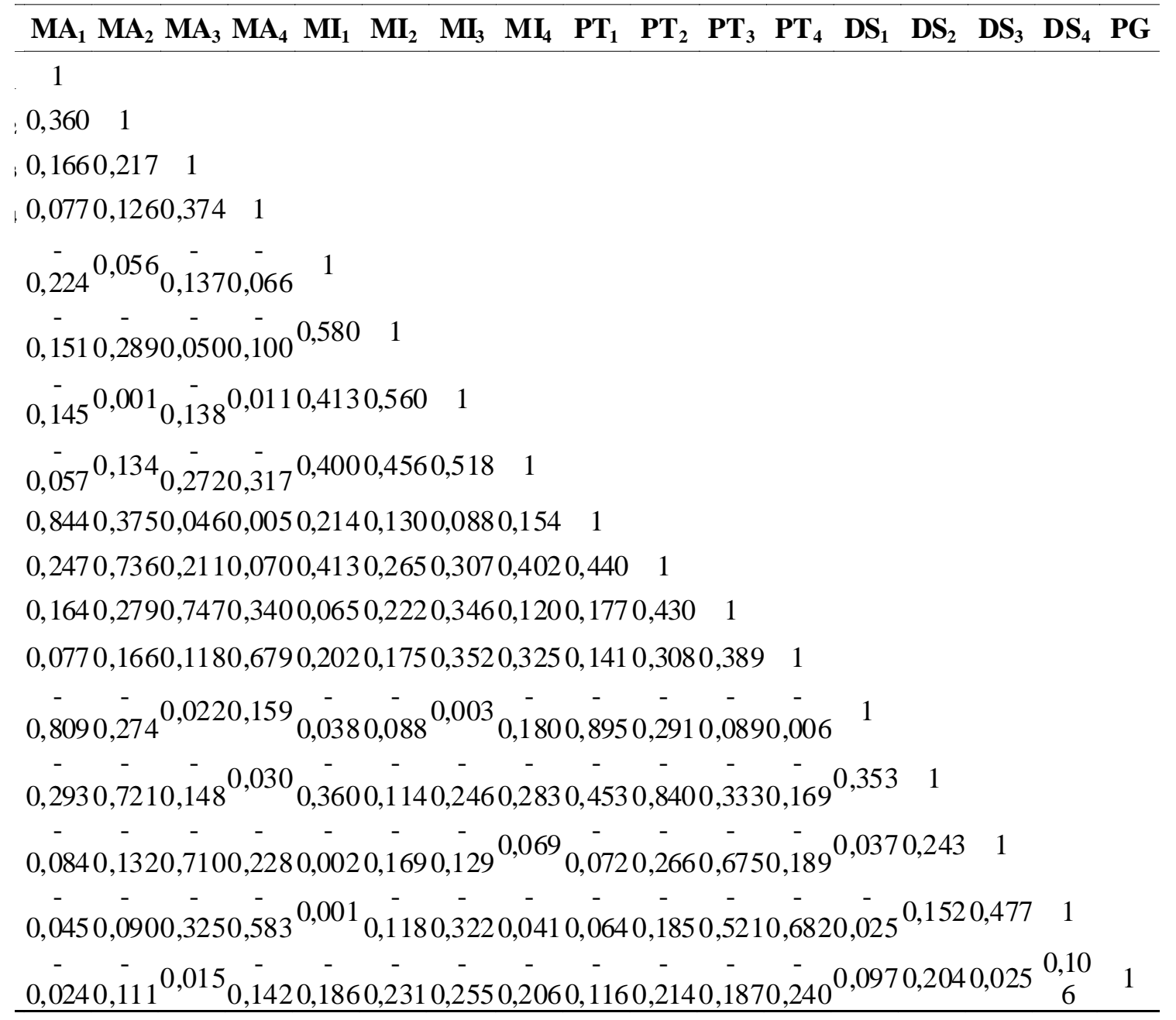


O maior e menor valor coeficiente de correlação foi de $-0,895\left(\mathrm{PT}_{1} \mathrm{xDS_{1 }}\right.$ ) e 0,001 $\left(\mathrm{MA}_{2} \mathrm{xMI} \mathrm{I}_{3}\right)$. Seguindo o critério de classificação do coeficiente de correlação (SHARP, s/d), os pares de atributos $\mathrm{PT}_{1} \times \mathrm{DS}_{1}(\mathrm{r}=-0,895), \mathrm{MA}_{1} \times \mathrm{PT}_{1}(\mathrm{r}=$ $0,844), \mathrm{PT}_{2} \times \mathrm{XS}_{2}(r=-0,840)$ e $\mathrm{MA}_{1} \times \mathrm{XS}_{1}(r=-$ $0,809)$ apresentaram as maiores correlações, classificadas como extra-altas. Já com correlações altas, apareceram os pares $\mathrm{MA}_{2} \times \mathrm{PT}_{2}$ $(r=0,736), \mathrm{MA}_{3} \times \mathrm{PT}_{3}(r=0,747), \mathrm{MA}_{2} \times \mathrm{XS}_{2}(r=-$ $0,721), \mathrm{MA}_{3} \times \mathrm{XS}_{3}(\mathrm{r}=-0,710), \mathrm{PT}_{4} \times \mathrm{XS}_{4}(\mathrm{r}=-0,682)$, $\mathrm{MA}_{4} \times \mathrm{PT}_{4}(\mathrm{r}=0,679)$ e $\mathrm{PT}_{3} \times \mathrm{XS}_{3}(r=-0,675)$. Os de moderada correlação foram $\mathrm{MA}_{4} \times \mathrm{DS}_{4}(\mathrm{r}=-$ $0,583), \mathrm{Ml}_{1} \times \mathrm{Ml}_{2}(r=0,580), \mathrm{Ml}_{2} \times \mathrm{Ml}_{3}(r=0,560)$, $\mathrm{PT}_{3} \mathrm{xDS} \mathrm{S}_{4}(\mathrm{r}=-0,521), \mathrm{Ml}_{3} \times \mathrm{XI}_{4}(\mathrm{r}=0,518), \mathrm{Ml}_{2} \mathrm{xMl_{4 }}$ $(r=0,456), P_{1} \times D S_{2}(r=-0,453), D_{3} \times D_{4}(r=$ $0,447), \mathrm{PT}_{1} \times \mathrm{PT}_{2}(r=0,440), \mathrm{PT}_{2} \times \mathrm{PT}_{3}(r=0,430)$, $\mathrm{Ml}_{1} \times \mathrm{Ml}_{3}(\mathrm{r}=0,413), \mathrm{Ml}_{1} \times \mathrm{PT}_{2}(\mathrm{r}=0,413), \mathrm{Ml}_{4} \times \mathrm{PT}_{2}$ $(r=0,402)$ e $\mathrm{MI}_{1} \times \mathrm{MI}_{4}(r=0,400)$. Dos 136 coeficientes de correlação apresentados naTabela 2, os 25 supracitados (4 extra-altos, 7 altos e 14 moderados) representam $18,4 \%$ dos dados, as demais correlações são classificadas comobaixas/nulas representando $81,6 \%$ das correlações.

A correlação linear entre os atributos físicos do solo $\left(\mathrm{MA}_{1}, \mathrm{MA}_{2}, \mathrm{MA}_{3}, \mathrm{MA}_{4}, \mathrm{Ml}_{1}, \mathrm{MI}_{2}, \mathrm{Ml}_{3}, \mathrm{MI}_{4}\right.$, $\mathrm{PT}_{1}, \mathrm{PT}_{2}, \mathrm{PT}_{3}, \mathrm{PT}_{4}, \mathrm{DS}_{1}, \mathrm{DS}_{2}, \mathrm{DS}_{3}$ e $\mathrm{DS}_{4}$ ) e a produtividade de grãos de feijão (PG) apresentou baixos/nulos valores de coeficiente de correlação, coincidindo com MEGDA et al. (2008).

Os parâmetros de ajuste do semivariograma são apresentados na Tabela 3. Verifica-se que $\mathrm{PT}_{2}, \mathrm{MA}_{3}, \mathrm{Ml}_{2}, \mathrm{Ml}_{3}, \mathrm{MI}_{4}$, e a $\mathrm{DS}_{2}$ apresentaram efeito pepita puro. A presença de efeito pepita puro indica que o espaçamento utilizado não foi suficiente para detectar a variabilidade espacial (SIQUEIRA et al., 2008). Os demais atributos de solo e planta se ajustaram a distintos modelos (esférico, exponencial e gaussiano), discordando de CAMBARDELLA et al. (1994), FREDDI et al. (2005), MEGDA et al. (2008), SIQUEIRA et al. (2008) que descrevem o modelo esférico como o que mais se ajusta aos parâmetros de solo e planta.

TABELA 3. Parâmetros de ajuste do semivariograma para os atributos em estudo

\begin{tabular}{|c|c|c|c|c|c|c|}
\hline Atributos & Modelo & $\mathrm{C}_{0}$ & $\mathrm{C}_{0}+\mathrm{C}_{1}$ & $\mathrm{C}_{1}$ & $\mathbf{a}(\mathbf{m})$ & RD $(\%)$ \\
\hline $\mathbf{P T}_{1}$ & Gaussiano & $2,00 \times 10^{-4}$ & $6,18 \times 10^{-4}$ & $4,18 \times 10^{-4}$ & 24,3 & 67,64 \\
\hline $\mathbf{P T}_{2}$ & \multicolumn{6}{|c|}{ Efeito pepita puro } \\
\hline $\mathbf{P T}_{3}$ & Exponencial & $1,89 \times 10^{-4}$ & $6,11 \times 10^{-4}$ & $4,22 \times 10^{-4}$ & 39,0 & 69,07 \\
\hline $\mathbf{P T}_{4}$ & Gaussiano & $1,65 \times 10^{-4}$ & $5,46 \times 10^{-4}$ & $3,81 \times 10^{-4}$ & 52,3 & 69,78 \\
\hline $\mathbf{M A}_{1}$ & Gaussiano & $1,15 \times 10^{-2}$ & $1,65 \times 10^{-2}$ & $5,01 \times 10^{-3}$ & 77,9 & 30,36 \\
\hline $\mathbf{M A}_{2}$ & Gaussiano & $9,50 \times 10^{-3}$ & $1,30 \times 10^{-2}$ & $3,50 \times 10^{-3}$ & 43,3 & 26,92 \\
\hline $\mathbf{M A}_{3}$ & \multicolumn{6}{|c|}{ Efeito pepita puro } \\
\hline $\mathbf{M A}_{4}$ & Gaussiano & $2,70 \times 10^{-4}$ & $4,00 \times 10^{-4}$ & $1,30 \times 10^{-4}$ & 46,7 & 32,50 \\
\hline $\mathbf{M I}_{1}$ & Esférico & $1,37 \times 10^{-4}$ & $2,76 \times 10^{-4}$ & $1,39 \times 10^{-4}$ & 70,2 & 50,36 \\
\hline $\mathbf{M I}_{2}$ & \multicolumn{6}{|c|}{ Efeito pepita puro } \\
\hline $\mathrm{MI}_{3}$ & \multicolumn{6}{|c|}{ Efeito pepita puro } \\
\hline $\mathbf{M I}_{4}$ & \multicolumn{6}{|c|}{ Efeito pepita puro } \\
\hline $\mathbf{D S}_{1}$ & Gaussiano & $3,70 \times 10^{-3}$ & $6,00 \times 10^{-3}$ & $2,30 \times 10^{-3}$ & 55,4 & 38,33 \\
\hline $\mathbf{D S}_{2}$ & \multicolumn{6}{|c|}{ Efeito pepita puro } \\
\hline $\mathbf{D S}_{\mathbf{3}}$ & Exponencial & $1,92 \times 10^{-3}$ & $3,85 \times 10^{-3}$ & $1,93 \times 10^{-3}$ & 71,7 & 50,13 \\
\hline $\mathbf{D S}_{4}$ & Esférico & $5,64 \times 10^{-4}$ & $2,55 \times 10^{-3}$ & $1,99 \times 10^{-3}$ & 71,5 & 78,04 \\
\hline PG & Esférico & $9,80 \times 10^{-3}$ & $5,85 \times 10^{-2}$ & $4,87 \times 10^{-2}$ & 22,9 & 83,25 \\
\hline
\end{tabular}


A produção de grãos (PG) e a densidade do solo na camada de 0,3-0,4 $\mathrm{m}$ de profundidade $\left(\mathrm{DS}_{4}\right)$ apresentaram forte razão de dependência espacial (RD, 83,25 \% e 78,04\% respectivamente). Os demais atributos apresentaram moderada razão de dependência espacial na seguinte ordem: $\mathrm{PT}_{4}>\mathrm{PT}_{3}>\mathrm{PT}_{1}>$ $\mathrm{MI}_{1}>\mathrm{DS}_{3}>\mathrm{DS}_{1}>\mathrm{MA}_{4}>\mathrm{MA}_{1}>\mathrm{MA}_{3}$. Esses valores foram relativamente iguais aos obtidos por SOUZA et al. (2001), CARVALHO et al. (2002), FREDDI et al. (2005) e SANTOS et al. (2005).

Com relação aos valores de alcance da dependência espacial $(a, m)$, a porosidade total (PT), macroporosidade (MA), microporosidade (MI) e a densidade do solo (DS) apresentaram

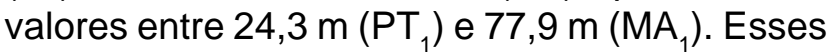
valores foram superiores aos encontrados por de SOUZA et al. (2001) e CARVALHO et al. (2002). A PG apresentou alcance de $22,9 \mathrm{~m}$, maior do que os valores de 6,1 e $10,0 \mathrm{~m}$, obtidos respectivamente por e SANTOS et al. (2005), assim como, menor do que o obtido por MEGDA et al. (2008) de 104,4 m.

Os mapas de variabilidade espacial (Figura 2) demonstram que $\mathrm{PT}_{1}(0,0-0,1 \mathrm{~m}$ de profundidade) apresenta um comportamento diferente de $\mathrm{PT}_{3}(0,2-0,3 \mathrm{~m}$ de profundidade) e $\mathrm{PT}_{4}$ (0,3-0,4 $\mathrm{m}$ de profundidade) na distribuição das linhas de contorno, concordando com a baixa correlação linear entre estes atributos (Tabela 2, $\left.\mathrm{PT}_{1} \times \mathrm{PT}_{3}=0,177 \mathrm{e} \mathrm{PT}_{1} \times \mathrm{PT}_{4}=0,141\right)$. $\mathrm{O}$ mesmo fato ocorre quando comparamos os mapas de macroporosidade do solo $\left(\mathrm{MA}_{1}, \mathrm{MA}_{2}\right.$ e $\left.M A_{4}\right)$, cujos valores de correlação linear é baixo para $\mathrm{MA}_{1} \times M A_{2}$ $(r=0,360)$ e nulo entre $\mathrm{MA}_{1} \times \mathrm{MA}_{4}(r=0,077)$. O mapa de $\mathrm{MI}_{1}$ apresenta similaridade na distribuição das linhas de contorno entre $\mathrm{PT}_{1} \mathrm{e}$ $\mathrm{MA}_{1}$, mesmo apresentando baixos valores de coeficiente de correlação.

A distribuição das linhas de contorno nos mapas de variabilidade espacial da densidade do solo (DS) demonstra que haver determinada relação espacial entre estes atributos. Quando comparamos os mapa de variabilidade espacial da produção de grãos de feijão (PG) e os mapas dos atributos físicos do solo (PT, MA, MI e DS) percebe-se que as zonas de variabilidade com maior produtividade não apresentam similaridade com os mapas dos atributos físicos, coincidindo com a baixa/nula correlação linear encontrada entre estes atributos.

\section{CONCLUSÕES}

A porosidade total (PT), macroporosidade (MA), microporosidade (MI), densidade do solo (DS) e a produtividade de grãos de feijão (PG) apresentaram baixa variabilidade. No geral, os atributos estudados não variaram aleatoriamente. Seguiram padrões espaciais bem definidos, com alcances da dependência espacial entre 22,9 e 77,9 metros; A correlação linear entre os atributos do solo variou entre nula a alta. A correlação linear entre os atributos do solo variou entre nula a alta. Entre a produtividade de grãos e os atributos físicos do solo a correlação linear foi nula. 
$\mathrm{PT}_{1}$

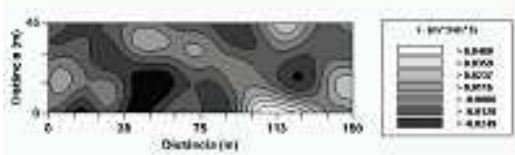

$\mathrm{MA}_{1}$

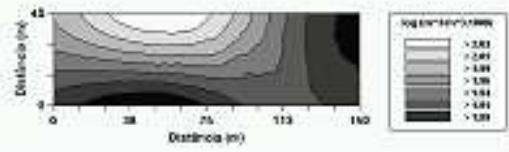

$\mathrm{MI}_{\mathrm{I}}$

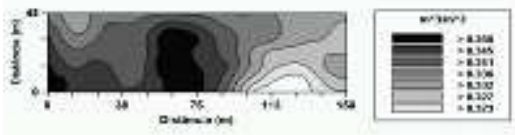

$\mathrm{DS}_{4}$

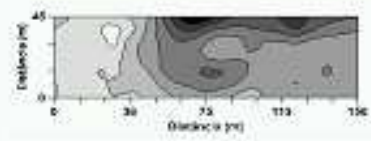

$\mathrm{PT}_{3}$

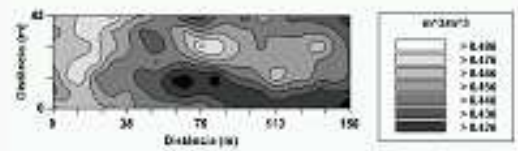

$\mathrm{MA}_{2}$

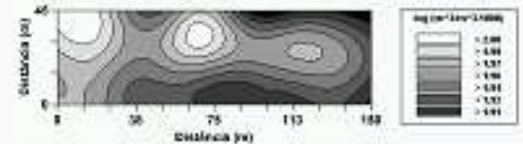

$D S_{1}$
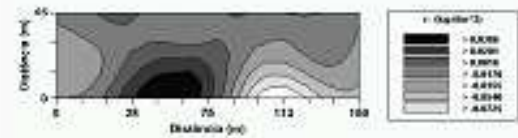

$\mathrm{PT}_{4}$

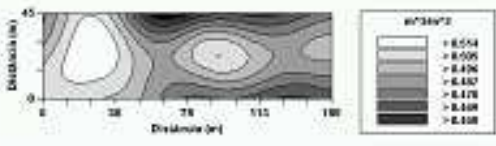

$\mathrm{MA}_{\mathrm{A}}$

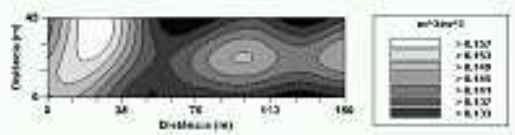

$\mathrm{DS}_{3}$

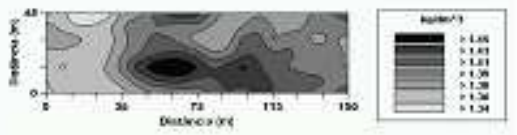

PG

FIGURA 2. Mapas de variabilidade espacial para os atributos físicos do solo $\left(\mathrm{PT}_{1} ; \mathrm{PT}_{3}, \mathrm{PT}_{4} ; \mathrm{MA}_{1}, \mathrm{MA}_{2}\right.$, $\mathrm{MA}_{4}, \mathrm{MI}_{1}, \mathrm{DS}_{1}, \mathrm{DS}_{3}$ e $\mathrm{DS}_{4}$ ) e de planta (PG) que apresentaram variabilidade espacial.

\section{REFERÊNCIAS BIBLIOGRÁFICAS}

ALCÂNTARA, E.N.; FERREIRA, M.M. Efeitos de métodos de controle de plantas daninhas na cultura do cafeeiro (Coffea arábica L.) sobre a qualidade física do solo. Revista Brasileira de Ciência do Solo, Viçosa, MG, v.24, p.711-721, 2000.

BAVER, L.D. Física de suelos. México: Unión Tipográfica Editorial Hispano-Americana., 1973, $529 p$.

BENGOUGH, A.G.; CAMPBELL, D.J.; O' SULLIVAN, M. F. Penetrometer techniques in relation to soil compaction and root growth. In: SMITH, K.A.; MULLINS, C.E., (Eds) Soil and environmental analysis: physical methods. 2 ed. New York: Marcel Dekker, 2001. 403.
BEUTLER, A.N.; SILVA, M.L.N; CURI, N.; FERREIRA, M.M.; CRUZ, J.C.; PEREIRA FILHO, I.A. Resistência à penetração e permeabilidade de Latossolo Vermelho distrófico típico sob sistemas de manejo na região dos cerrados. Revista Brasileira de Ciência do Solo, Viçosa, MG, v.25, p.167-177, 2001.

BOLLER, W.; CALDATO, D. Desenvolvimento da cultura do feijão (Phaseolus vulgaris L.) em diferentes condições de cobertura e de preparo do solo. Energia na agricultura, Sorocaba, SP, v.21, n.2, p.167-173, 2001.

CAMBARDELLA, C.A.; MOORMAN, T.B.; NOVAK, J.M.; PARKIN, T.B.; KARLEN, D.L.; TURCO, R.F.; KONOPKA, A.E. Field-scale variability of soil properties in Central lowa Soils. 
Soil Science Society of America Journal, Madison, WI, v.58, p.1501-1511, 1994.

CARVALHO, M.P.; SORATTO, R.P.; FREDDI, O.S. Variabilidade espacial de atributos físicos em um Latossolo Vermelho Distrófico sob preparo convencional em Selvíria (MS). Acta Scientiarum, Maringa, PR, v.24, p.1353-1361, 2002.

EMPRESA BRASILEIRA DE PESQUISA AGROPECUÁRIA - EMBRAPA Manual de métodos de análise de solos. 2.ed. Rio de Janeiro: Serviço Nacional de Levantamento e Conservação do Solo.,1997. 212p.

EMPRESA BRASILEIRA DE PESQUISA AGROPECUÁRIA-EMBRAPA. Sistema brasileiro de classificação de solos. Rio de Janeiro, 2006. $306 p$.

FAHL, J.I; CAMARGO, M.B.P.; PIZZINATTO, M.A; BETTI, J.A.; MELO, A.M.T.; DEMARIA, I.C.; FURLANI, A.M.C. Instruções agrícolas para as principais culturas econômicas. 6. ed. Campinas: Instituto Agronômico, 1998. p. 281282. (Boletim Técnico, 200).

FREDDI, O.S.; CARVALHO, M.P.; CENTURION, J.F.; BARBOSA, G.F. Variabilidade espacial da produtividade do feijão em um Latossolo Vermelho distroférrico sob preparo convencional e plantio direto. Acta Scientiarum, Maringa, PR, v.27, p.61-67, 2005.

GOLDEN SOFTWARE. Surfer for Windows, version 7.0: Software de geração de mapas de contorno e superfícies contínuas. Colorado, 1999.

GOMES, F.P., GARCIA, C.H. Estatística aplicada a experimentos agronômicos e florestais. Piracicaba: FEALQ, 2002. 309p.

KIEHL, E. J. Manual de edafologia: relações soloplanta. Sao Paulo: Editora Agronômica Ceres Ltda. 1979, 264p.

MEGDA, M.M.; CARVALGO, M.P.; VIEIRA, M.X.; ANDREOTTI, M.; PEREIRA, E.C. Correlação linear e espacial entre a produtividade de feijão e a porosidade de um Latossolo de Selvíria (MS).
Revista Brasileira de Ciência do Solo, Viçosa, MG, v.32, p.781-788, 2008.

NEHMI, I.M.D. et al. Feijão In: NEHMI, I. M. D. et al. AGRIANUAL 2003: Anuário da Agricultura Brasileira. São Paulo: Argos, 2001. p.329-336. (AGRIANUAL, 2003).

ROBERTSON, G.P. GS+: Geostatistics for the environmental sciences. Michigan: Gamma Desing Software, 1998. 151p.

SANTOS, P.A.; CARVALHO, M. P.; FREDDI, O.S.; KITAMURA, A.E.; FREITAG, E.E.; VANZELA, L.S. Correlação linear e espacial entre o rendimento de grãos do feijoeiro e a resistência mecânica à penetração em um Latossolo Vermelho Distrófico. Revista Brasileira de Ciência do Solo, Viçosa, MG, v.29, p.287-295, 2005.

SCHLOTZHAVER, S.D.; LITTELL, R.C. SAS: system for elementary statistical analysis. 2 . ed. Cary: SAS, 1997. 441p.

SHAPIRO, S.S.; WILK, M.B. An analysis of variance test for normality: complete samples. Biometrika, London, UK, 52: 591-611, 1965.

SHARP, s/d. Instructor manual. Scientific computer, Model EL-5500 II. Japan: Sharp Eletronics Corporation , 199?, 196p.

SIQUEIRA, G.M.; VIEIRA, S.R.; CEDDIA, M.B. Variabilidade espacial de atributos físicos do solo por métodos diversos. Bragantia, Campinas, SP, v.67, n.1, p.203-211, 2008.

SOUZA, Z. M.; MARQUE JÚNIOR, J. \& PEREIRA, G. T. Variabilidade espacial de atributos físicos do solo em diferentes formas do relevo sob cultivo de cana-de-açúcar. Revista Brasileira de Ciência do Solo, Viçosa, MG, v.28, p.937-944, 2004.

SOUZA, Z.M.; SILVA, M.L.S.; GUIMARÃES, G.L.; CAMPOS, D.T.S.; CARVALHO, M. P.; PEREIRA, G.T. Variabilidade espacial de atributos físicos em um Latossolo Vermelho distrófico sob semeadura direta em Selvíria (MS). Revista Brasileira de Ciência do Solo, Viçosa, MG, v.25, p.699-707, 2001. 
TRANGMAR, B. B.; YOST, R. S.; UEHARA, G. Application of geostatistics to spatial studies of soil properties. Advances in Agronomy, Newark, NJ, v.38, p.45-94, 1985.

VIEIRA, S. R. Variabilidade espacial de argila, silte e atributos químicos em uma parcela experimental de um Latossolo Roxo de Campinas (SP). Bragantia, Campinas, SP, v.56, p.181-190, 1997.

WEBSTER, R.; OLIVER, M. A. Statistical methods in soil and land resource survey. Oxford: Oxford University Press, 1990. 316p. 